\title{
Ferroptosis in Rheumatoid Arthritis: A Potential Therapeutic Strategy
}

\author{
Ting Zhao ${ }^{\dagger}$, Qi Yang ${ }^{\dagger}$, Yujiang Xi ${ }^{\dagger}$, Zhaohu Xie, Jiayan Shen, Zhenmin Li, Zhaofu Li ${ }^{*}$ \\ and Dongdong Qin
}

School of Basic Medical Sciences, Yunnan University of Chinese Medicine, Kunming, China

OPEN ACCESS

Edited by:

Valentina Pucino,

University of Birmingham,

United Kingdom

Reviewed by:

Chris Wincup,

University College London,

United Kingdom

Federico Pratesi,

University of Pisa, Italy

${ }^{*}$ Correspondence:

Zhaofu Li

Izf0817@126.com

Dongdong Qin

qindong108@163.com

${ }^{\text {t}}$ These authors have contributed equally to this work

Specialty section: This article was submitted to Autoimmune and

Autoinflammatory Disorders,

a section of the journal

Frontiers in Immunology

Received: 19 September 2021 Accepted: 14 January 2022 Published: 02 February 2022

Citation:

Zhao $T$, Yang $Q, X i$ Y, Xie Z, Shen J, Li Z, Li Z and Qin D (2022) Ferroptosis in Rheumatoid Arthritis: A Potential Therapeutic Strategy.

Front. Immunol. 13:779585. doi: 10.3389/fimmu.2022.779585
Ferroptosis is one of the newly discovered forms of cell-regulated death characterized by iron-dependent lipid peroxidation. Extensive research has focused on the roles of ferroptosis in tumors, blood diseases, and neurological diseases. Some recent findings have indicated that ferroptosis may also be related to the occurrence and development of inflammatory arthritis. Ferroptosis may be a potential therapeutic target, and few studies in vitro and animal models have shown implications in the pathogenesis of inflammatory arthritis. This mini review discussed the common features between ferroptosis and the pathogenesis of rheumatoid arthritis (RA), and evaluated therapeutic applications of ferroptosis regulators in preclinical and clinical research. Some critical issues worth paying attention to were also raised to guide future research efforts.

Keywords: ferroptosis, rheumatoid arthritis, lipid peroxidation, reactive oxygen species, therapeutic strategy

\section{INTRODUCTION}

Rheumatoid arthritis (RA) is the most specific systemic immune system disease among autoimmune diseases (1), invading many joints, such as knee and elbow joints. Its main clinical manifestations are joint swelling and stiffness in the morning. RA has an incidence of $0.5 \%$ to $1 \%$, with an apparent reduction from north to south (in the northern hemisphere) and from urban to rural areas (2). Some Native American populations have a very high prevalence. The incidence of RA is high in 30-50 years of age, and the incidence in women is about three times that of men. RA may be related to various cell types and cytokines (3), and the origin of its pathology is autoantibodies $(4,5)$. RA is characterized by infiltration of macrophages and lymphocytes, the proliferation of synovial fibroblasts, joint inflammation, progressive cartilage destruction, and bone erosion, as well as degenerative manifestations (6).

Disease-modifying antirheumatic drugs (DMARDs) are conventional drugs in the treatment of RA. Depending on the symptom severity, RA can be treated with a single drug or a combination of 2,3 , or 4 drugs $(7,8)$. When the disease is refractory, some biologic DMARDs are recommended. The use rate of biological agents in the treatment of RA in North America has been as high as 50.7\% (9). However, the clinical efficacy rate is inconsistent, ranging from $50 \%$ to $70 \%(10)$. In clinical practice, we should make changes in the therapeutic strategy when the arthritis is resistant to initial therapy. Hence, there is an urgent need to develop drugs with new targets or new mechanisms of action to meet the clinical needs of patients.

In RA, mature $\mathrm{B}$ cells and dendritic cells present antigens to $\mathrm{T}$ cells, leading to $\mathrm{T}$ cell activation. Different immune cells secrete unique cytokines and jointly stimulate the expression of cytokine 
TRANCE/receptor activator of nuclear factor-kappa B ligand (RANKL), which is necessary for osteoclast differentiation. B-T cell interaction leads to the activation of plasma cells responsible for producing and secreting autoantibodies. Autoantibodies, cytokines, and RANKL stimulate osteoclasts to cause bone resorption and induce cartilage damage driven by chondrocytes. In addition, compared with activated B cells, transitional B cells can inhibit the formation of osteoclasts in an immunomodulatory manner by providing IL-10. It is reported that ferroptosis plays a vital role in the occurrence and development of many diseases such as Parkinson's disease, ischemia-reperfusion injury, and tumors $(11,12)$. Recent studies have shown that ferroptosis plays a critical regulatory role in autoimmune and inflammatory diseases $(13,14)$. New strategies for targeting ferroptosis are to regulate the immune response homeostasis, and in some cases, the reactions can influence each other. Studies have found that ZIP14, a ferroptosis-related metal transporter, may play a regulatory role in the immune system (15). Early studies have confirmed that glutathione peroxidase (GPX) activity in polymorphonuclear leucocytes of RA patients with high persistent disease activity is reduced (16). Luo et al. (17) found that RSL3, a ferroptosis activator, can induce ferroptosis in synovial cells and aggravate synovitis. Transferrin receptor 1 (TFR1) and nuclear receptor coactivator 4 (NCOA4) were upregulated, but system Xc- (an amino acid transporter mediating the exchange of extracellular cystine and intracellular glutamate) and GSH-glutathione peroxidase 4 (GPX4), as well as nuclear factor erythroid 2-related factor 2 (Nrf2, a transcriptional factor that induces antioxidative and cytoprotective responses), were downregulated by RSL3 treatment. Herein, ferroptosis may be a potential therapeutic target for inflammatory arthritis in the future.

This mini review discussed the common features between ferroptosis and the pathogenesis of RA, and evaluated therapeutic applications of ferroptosis regulators in preclinical and clinical research. Some critical issues worth paying attention to were also raised to guide future research efforts.

\section{FERROPTOSIS IN CELL DEATH}

Cell death is a sophisticated process, and its mechanisms have traditionally been divided into two types, programmed cell death (PCD) mechanisms that require energy, and necrotic cell death mechanisms that do not (18). In addition, necrotic cell death typically causes a strong immune response, whereas PCD does not $(19,20)$. In 2012, Dixon et al. (21) discovered a unique iron-dependent form of nonapoptotic cell death when studying the mechanism of the small molecule compound (named erastin) against RAS mutant tumors, which was called ferroptosis. It is significantly different from other death patterns in morphology, biochemistry, and genetics (22). It is characterized by the accumulation of lethal reactive oxygen species (ROS) arised from the reaction between iron and lipid peroxides, which are themselves generated by the oxidation of polyunsaturated fatty acids (PUFAs)-containing phospholipids (PUFA-PLs) (23). PUFAs are essential for ferroptosis due to their sensitivity to lipid peroxidation (24). Free PUFAs are involved in ferroptosis after they are esterified into PUFA-PL and PUFA-PLOOH. Ferroptosis does not have morphological characteristics of apoptosis, such as cell shrinkage, chromatin agglutination, formation of apoptotic bodies, disintegration of cytoskeleton, and other phenomena. However, it can be observed that the volume of mitochondria decreases and the membrane's density increases (25), which are not observed in apoptosis. At the same time, along with mitochondrial morphology alterations accompanying ferroptosis, a common morphological feature is cell ballooning/blistering followed by plasma membrane rupture (26). In terms of biochemical characteristics, ferroptosis is mainly triggered by glutathione (GSH) depletion and glutathione peroxidase 4 (GPX4) inactivation. It is mainly related to lipid peroxidation metabolism and intracellular iron balance, and several genes are involved in the regulation of ferroptosis. Ferroptosis inducers mainly include erastin, FINO2, and RSL3. Ferroptosis inhibitors mainly include liproxstatin-1, iron chelator, and ferrostatin-1. Liproxstatin-1 free radicals, which were formed by removing lipid peroxides from liproxstatin-1, can be reduced by other antioxidants (such as ubiquinone). Zika et al. found that liproxstatin-1 may reduce the accumulation of intracellular toxic lipid ROS, thereby inhibit the occurrence of cell ferroptosis (27). At present, the understanding of ferroptosis is not comprehensive enough, and its mechanism is still in the exploratory stage.

\section{THE POTENTIAL ROLE OF FERROPTOSIS IN RA}

Ferroptosis might play a role in the onset of RA and may be used as a treatment option in the future. Recently, it has been found that RA and ferroptosis have similar characteristics, mainly in the following aspects.

\section{IRON HANDLING}

Abnormal iron metabolism is an important cause of ferroptosis. Regulatory pathways of intracellular iron homeostasis mainly include ferroportin and TFR1 to regulate iron export and absorption (23). Iron ions induce the body to produce a large amount of lipid ROS through fenton reaction, promote lipid peroxidation, and lead to ferroptosis. Under oxidative stress conditions, superoxide will be produced in a short time, reducing $\mathrm{Fe}^{3+}$ stored in ferritin to $\mathrm{Fe}^{2+}$, resulting in the release of iron ions. $\mathrm{Fe}^{3+}$ enters the cell under the transport of TFR1, and then is converted into $\mathrm{Fe}^{2+}$. The excess iron ions are stored in ferritin to control the storage of iron ions. In addition, both iron response element binding protein 2 (IREB2) and Nrf2 are involved in regulating $\mathrm{Fe}^{2}+$ in cells. NCOA4 recognizes and relies on the autophagy pathway to degrade intracellular ferritin, releasing free iron ions (28) (Figure 1). 


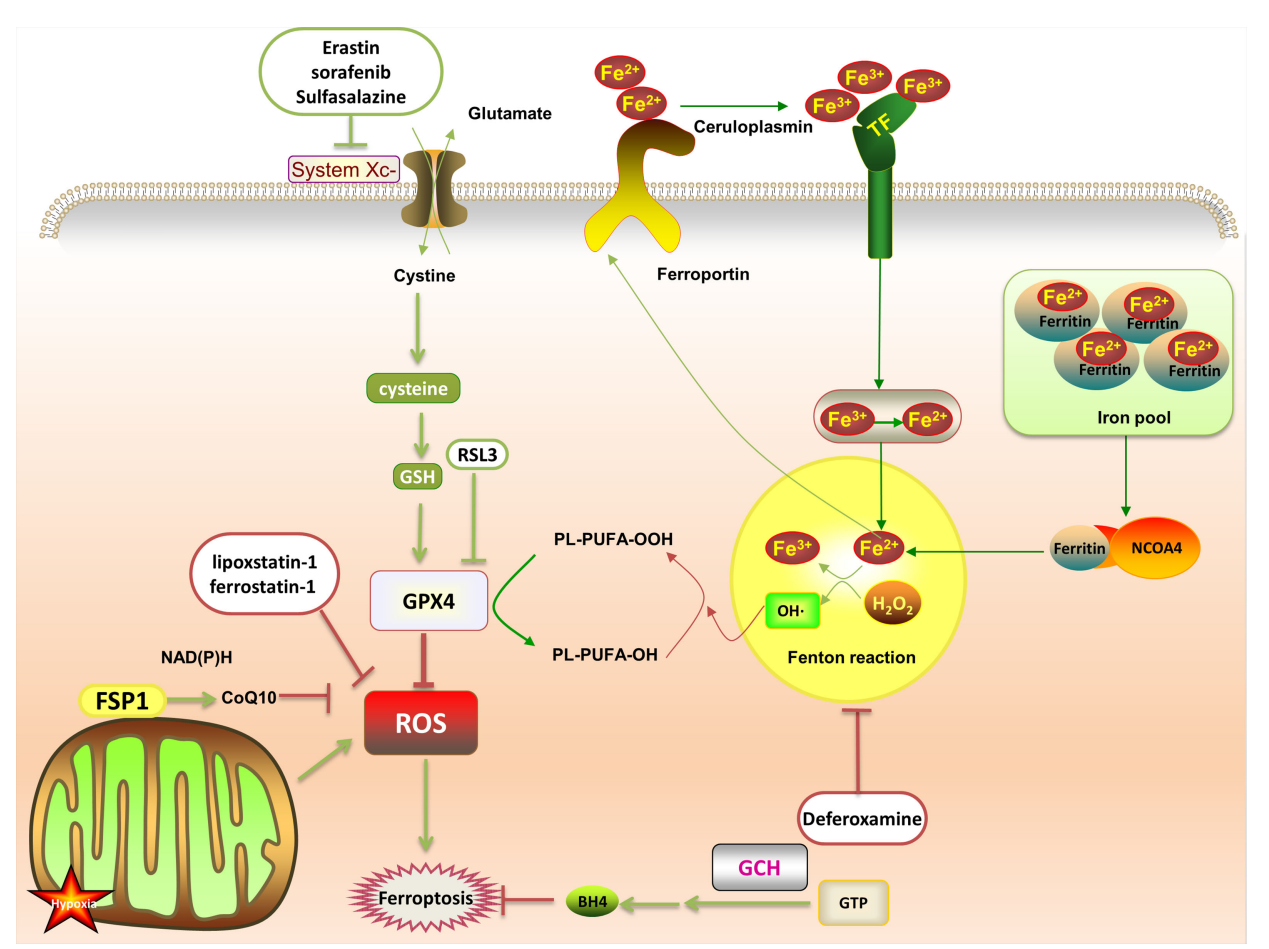

FIGURE 1 | After the transferrin binds to the transferrin receptor on the plasma membrane, the plasma membrane forms a vesicle that takes $\mathrm{Fe}^{3+}$ carrying transferrin into the cell. Then, the low pH in the vesicle promotes the separation of $\mathrm{Fe}^{3+}$ from the transferrin and the shedding of $\mathrm{Fe}^{3+}$. It is reduced to $\mathrm{Fe}^{2+}$ and free in the cytoplasm, or is combined with ferritin to form an iron pool. The feritin in the iron pool can be encapsulated by autophagy lysosomes under the mediation of NCOA4, and then degraded and release a large amount of $\mathrm{Fe}^{2+}$. $\mathrm{Fe}^{2+}$ and $\mathrm{H}_{2} \mathrm{O}_{2}$ generate $\mathrm{PLOOH}$ through the fenton reaction, which promotes ferroptosis by promoting further lipid peroxidation and selfperoxidation. In the GSH/GPX4 pathway, with the help of GSH, GPX4 down-regulates ROS and inhibits ferroptosis. This can be suppressed by RSL3. System Xc- (cystine/ glutamate antiporter) promotes synthesis of glutathione, which can be offset by erastin, sulfasalazine and sorafenib. In the FSP1 protection pathway, FSP1 can catalyze the reduction of CoQ10 to panthenol and consume $\mathrm{NAD}(\mathrm{P}) \mathrm{H}$ to inhibit ROS. In the $\mathrm{GCH} 1$ protection pathway, $\mathrm{GCH} 1$ acts as a rate-limiting enzyme to manage the biosynthesis of $\mathrm{BH} 4$ and reduce ferroptosis.

The proliferation and activation of osteoclasts lead to RA bone damage and bone metabolism disorders. In vitro and in vivo studies have shown that iron overload can induce osteoclast differentiation and inhibit osteoblast proliferation by increasing ROS generation (29). Low concentrations of iron ions can promote the growth of osteoblast precursor cells (MC3T3-E1), while high concentrations of iron ions inhibit their growth and increase ROS levels (30). Iron overload can inhibit the activity of osteoblasts to a certain extent, thereby affect their differentiation process. Simultaneously, it can also activate the differentiation of osteoclasts and cause bone destruction (31). Studies have shown that excessive iron ions can activate p38-MAPK and block PI3K/ AKT and JAK/STAT3 signaling pathways to induce MC3T3-E1 cell death (30). In addition, iron ions initiate the growth of synovial pannus by regulating the expression of critical genes such as $c-m y c$ and $m d m 2$. These genes are responsible for the proliferation of synovial cells and promote the occurrence and development of vascular synovitis (32).

Studies have demonstrated that iron deposits are found in osteoarthritis and RA $(33,34)$. Both osteoarthritis and rheumatoid synovia contained iron, but in the latter greater quantities were present. However, none of the controls with normal synovia had iron deposition. Another study found that the iron metabolism is different in RA than in general health (35). It is worth noting that iron deficiency is common (64\%) in RA patients with high disease activity. RA patients had lower hepcidin, lower transferrin, and lower ferritin. Icariin has antibacterial, anti-inflammatory and antioxidant effects (36). It has been suggested that icariin counteracts the effects of RSL3 on iron content, lipid peroxidation, and relative protein (SLC7A11, SLC3A2L, GPX4, TRF, NCOA4, and Nrf2) in synoviocytes given the observation that icariin might play a role in protecting synovial cells from ferroptosis (17). Herein, it can be exploited as a new therapeutic strategy for RA.

\section{MEMBRANE LIPID ANTIOXIDANT SYSTEM}

GSH is a tripeptide containing sulfhydryl groups combined with glutamic acid, cysteine, and glycine, which has an antioxidant effect. Under normal circumstances, the cystine entering the cell is reduced to cysteine to participate in the synthesis of GSH, which helps reduce the accumulation of lipid peroxides. However, when system Xc- is inhibited, GSH synthesis disorder will promote the 
decline of cellular antioxidant capacity and the accumulation of lipid ROS. Abnormally elevated lipid ROS levels can be controlled by GPX4 (37). GPX4 can effectively repair the oxidative damage of unsaturated fatty acids in mammals, thereby inhibiting ferroptosis. Stockwell et al. determined that the GPX4/GSH axis and the system Xc- regulate ferroptosis, considered a classic pathway (38). Earlier studies have shown that erastin, a system Xc- inhibitor, inhibits GSH synthesis and increases lipid ROS, leading to ferroptosis (21). Erastin has been shown to contribute to cartilage tissue damage by promoting matrix metalloproteinase 13 (MMP-13) expression and inhibiting type II collagen expression in chondrocytes (39), which may aggravate RA.

In addition to GPX4/GSH axis, ferroptosis suppressor protein 1 (FSP1)- Coenzyme Q10 (CoQ10) pathway has also been found to be related to ferroptosis. GPX4 and FSP1 are two parallel membrane lipid antioxidant pathways. FSP1 is one of the CoQ10 oxidoreductases, most of which are attached to the outer mitochondrial membrane (40). The FSP1-CoQ10 pathway can improve lipid peroxidation through free radical capture, and the process of ferroptosis is also blocked. Some studies speculate that FSP1 may improve RA through the TNF- $\alpha /$ ROS positive feedback loop $(41,42)$. However, the specific contribution of ferroptosis as a mode of cell death was not addressed in these studies.

It is worth noting that the GTP cyclohydrolase-1 (GCH1)tetrahydrobiopterin $(\mathrm{BH} 4)$ pathway is parallel but independent of the GPX4 and FSP1 pathways (43). GCH1 is the primary ratelimiting enzyme for the synthesis of $\mathrm{BH} 4$. Overexpression of $\mathrm{GCH} 1$ can enhance the production of $\mathrm{BH} 4$, and then reduce ferroptosis. So far, few such evidence has been provided for RA. Although the GCH1-BH4 protective pathway is closely related to ferroptosis, the interaction between GCH1-BH4 and RA is still not fully understood, and further research is needed.

\section{OXIDATIVE STRESS AND LIPID PEROXIDATION}

Studies have shown that oxidative stress plays a vital role in the progression of RA. ROS, as a product of oxidative stress, exists in the articular cavity of RA patients in large quantities. ROS can be used as a potential marker for the progression of RA patients (44). Excess ROS can be converted to hydrogen peroxide through the fenton reaction. In this process, $\mathrm{Fe}^{3+}$ can be reduced to $\mathrm{Fe}^{2+}$, generating hydroxyl (-OH) or alkoxy ( $\mathrm{RO}-)$ free radicals, and causing cell ballooning/blistering followed by plasma membrane rupture (45). ROS in cells can activate the NLRP3 receptor protein. Activated NLRP3 is polymerized by ATP to form highly ordered NLRP3 protein oligomers. Under the action of ASC, NLRP3 and pro-caspase-1 are connected to form a complex pro-caspase-1 that can be activated to form an enzymatic activity. The heterodimer caspase- 1 cuts the inactive pro-IL-1 $\beta$ and pro-IL-18 into mature IL-1 $\beta$ and IL-18, aggravating RA. When the local inflammation of RA joints accelerates, it can be used as an endogenous signal regulator to expand the synovial inflammation response (46). ROS is a key element of the ROS/TNF- $\alpha$ feedback loop. The production of TNF- $\alpha$ depends on the activation of NF- $\kappa B$ signaling pathway stimulated by ROS, which in turn activates the p38/JNK signaling pathway to accelerate the progression of RA (41) (Figure 2). ROS can induce activation of metalloproteinases, inhibit the synthesis of cartilage proteoglycans, and promote chondrocyte apoptosis, which eventually leads to cartilage destruction and bone erosion. This is consistent with pathogenic manifestations of RA. A study detected a strong positive correlation between the ROS level and the severity score in RA patients (47). The levels of lipid peroxidation in the serum and synovial fluid are increased in RA patients, and the antioxidant system has also changed (48). Hence, ROS production in excess is more likely to inhibit osteoblast differentiation and lead to bone destruction.

In addition, studies have found that ROS production can also be induced by activation of the phagocyte NADPH oxidase 2 (NOX2) complex in a process generally referred to as an oxidative burst. NOX2-derived ROS have been shown to suppress antigen-dependent $\mathrm{T}$-cell reactivity and remarkably to reduce the severity of experimental arthritis in both rats and mice (49). In addition, NOX2 also plays a role in antigen presentation and regulation of adaptive immunity. In $\mathrm{CD} 4^{+} \mathrm{T}$ cells, the lack of NOX2 induces the production of Th17 cells and reduces regulatory $\mathrm{T}$ cells in a ROS-dependent manner by affecting Foxp3 and ROR $\gamma$ t (50). The immunosuppressive properties of $\mathrm{CD} 4^{+} \mathrm{CD} 25^{+}$Foxp $^{+}$Treg cells play a vital role in maintaining the body's immune tolerance and immune response homeostasis. Early studies have demonstrated that regulatory $\mathrm{T}$ cells are functionally compromised in RA (51). The $\mathrm{CD} 4^{+} \mathrm{CD} 25^{+}$Treg cells in the joint synovial fluid of RA patients are significantly increased (52). In addition, the reduction of NOX2 will increase Th1, Th2, and Th17 cells, leading to inflammatory arthritis. Antigen-presenting cells (APCs) are known to produce NOX2derived ROS. A study found that the NOX2-dependent processing of the redox-sensitive autoantigens by APCs modified $\mathrm{T}$ cell activity and induced development of RA in mouse models (53). Despite many unknown facts, drugs targeting ferroptosis may represent a potential strategy for treating RA.

\section{INFLAMMATION}

Ferroptosis can also trigger the body's innate immunity, release inflammatory mediators, and activate the body's inflammatory response (14). Changes in the synovial membrane's typical physiological and metabolic properties can produce many inflammatory mediators, such as IL- $1 \beta$, TNF- $\alpha$, and IL-6, which increase uptake of transferrin and non-transferrinbound iron by monocytes and increase the uptake of transferrin-bound iron by synovial fibroblast (54). Increased iron intake accelerates the vicious cycle of hemorrhagesynovitis-hemorrhage, and the proliferated synovial tissue spreads to the surface of the articular cartilage. Cartilage matrix is degraded by connective cathepsin released by 


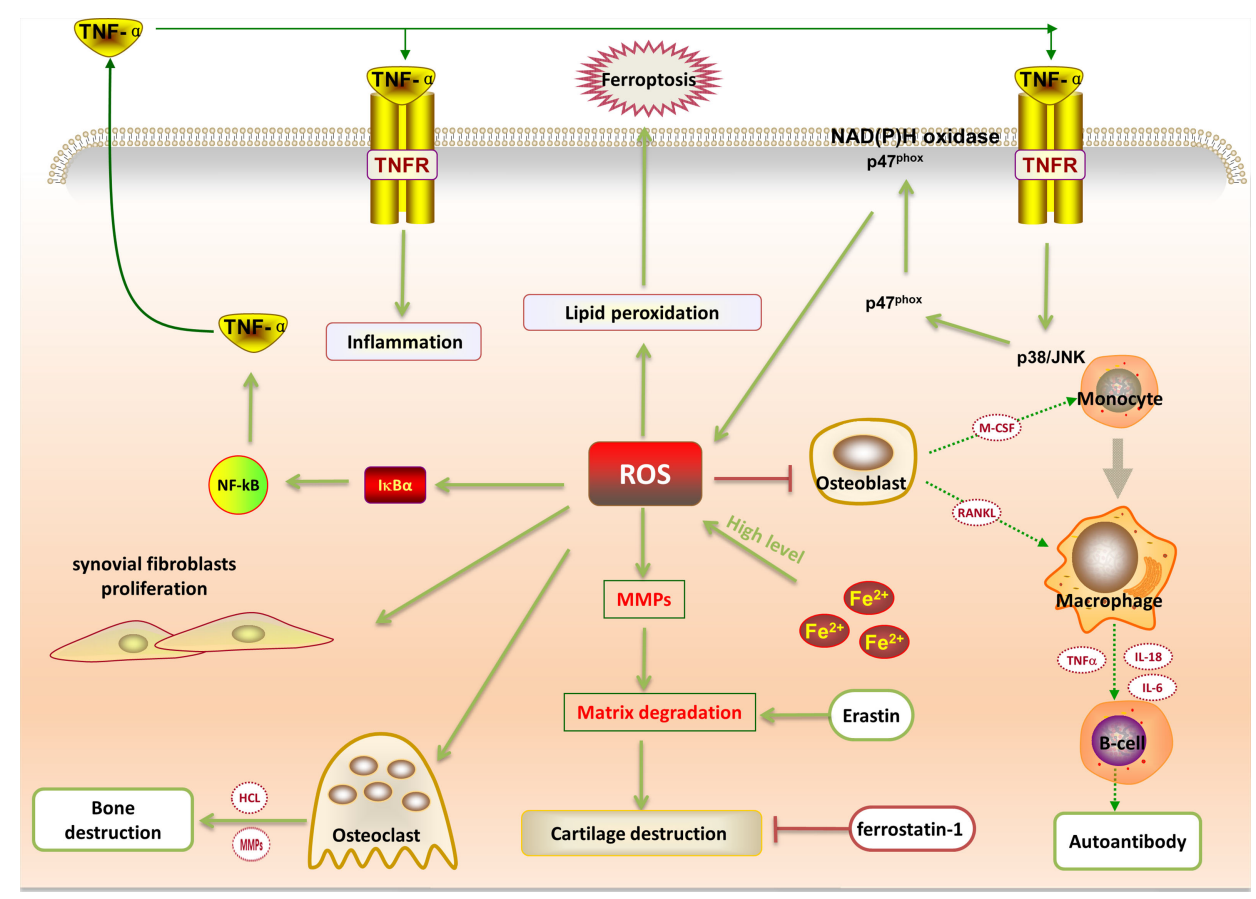

FIGURE 2 | ROS is a critical element of the ROS/TNF- $\alpha$ feedback loop. The production of TNF- $\alpha$ depends on the activation of ROS-stimulated NF-kB signaling, which activates the p38/JNK signaling pathway to accelerate the progression of RA inflammation. High levels of iron ions can catalyze the production of ROS. Excessive ROS will aggravate the proliferation of synovial fibroblasts; induce osteoclast differentiation and inhibit osteoblast proliferation; activate metalloproteinases, as well as lead to cartilage destruction and bone erosion. Excessive ROS will also promote lipid peroxidation, leading to cell ferroptosis. Moreover, the ferroptosis inducer (erastin) can promote the expression of matrix metalloproteinase 13 and promote cartilage destruction, while the ferroptosis inhibitor ferrostatin-1 can reduce cartilage degradation.

hypertrophic synovial tissue, chondrocytes and intravascular tissue, which will eventually lead to the destruction of articular cartilage and bone $(55,56)$.

\section{FERROPTOSIS: A RISING STAR WITH GREAT THERAPEUTIC POTENTIAL IN RA}

P53 is an excellent tumor suppressor gene that can inhibit system Xc- uptake of cystine by down-regulating the expression of SLC7A11 and result in a decrease in antioxidant capacity. Previous studies have confirmed that p53 protein is expressed in RA fibroblast-like synovial cells, and its overexpression is a characteristic of RA (57). It plays a role in controlling the balance between Th17 cells and Treg (58). Aberrant p53/p21 activationmediated aging-related secretory phenotype can accelerate destruction of cartilage tissue (59). Studies have shown that endogenous p53, which is inducible in rheumatoid synovial cells, is functionally active based on the findings that its expression blocks the G1/S transition by inhibiting the CDK-mediated phosphorylation of $\mathrm{Rb}$ via p21 induction (60). Clinical studies have found that the expression of p53 in lymphocytes is lower in RA patients than that of healthy people $(61,62)$. Acyl-CoA synthetase long-chain family member 4 (ACSL4, an enzyme involved in the activation of PUFAs) is located in peroxisomes and mitochondria, which can determine the sensitivity of cells to ferroptosis activation. Doll et al. found GPX4-ACSL doubleknockout cells showed marked resistance to ferroptosis (63). Mechanistically, ACSL4 enriched cellular membranes with long polyunsaturated $\omega 6$ fatty acids. ACSL 4 has a marked preference for activating PUFAs (64), therefore, deletion of ACSL4 prevents PUFAs from being incorporated into membrane PLs where they would become oxidized following GPX4 inactivation (26). Clinical studies have found that the ACSL4 is down-regulated in RA patients (65). BECN1 is a crucial regulator of autophagy, which can promote ferroptosis by regulating the activity of the system Xc-. BECN1dependent ferroptosis requires the formation of BECN1-SLC7A11 complex. Studies have found that autophagy of osteoblasts affects bone metabolism, and BECN1 may become a new target for the treatment of bone metabolism diseases (66). CoQ10, a fat-soluble antioxidant, is a crucial regulator of ferroptosis. Studies have shown that CoQ10 has anti-inflammatory effects on autoimmune diseases. Jhun et al. used CoQ10-encoded liposome/gold hybrid nanoparticles targeting STAT3/Th17 to slow RA's progression (67). Although the FSP1-CoQ10 protective pathway is closely related to RA, the interaction between CoQ10 and other ferroptosis regulators is still not fully understood, and further research is needed.

In addition, many studies have focused on the relationship between oxidative stress metabolism and ferroptosis regulators. For example, Nrf2 and heme oxygenase-1 (HO-1) level can regulate 
ferroptosis (68). A study found that reduced levels of the Nrf2 factor can lead to RA (69). Targeted activation of Nrf2 can inhibit ROS production, which in turn inhibits the proliferation and migration of RA fibroblast-like synovial cells (70). Luo $\mathrm{H}$ et al. found that RSL3 can reduce Nrf2 and GPX4 in synovial cells (17). In addition, lack of Nrf2 can lead to changes in the expression of SLC7A11, which further leads to oxidative stress damage and aggravates joint destruction (71). Studies have found that ferroptosis can be induced through the Nrf2SLC7A11-HO-1 pathway, which may play a regulatory role in joint destruction $(71,72)$.

The study found that FDA-approved RA drugs such as sulfasalazine and auranofin can prevent cell growth and induce ferroptosis. Sulfasalazine and auranofin activity were largely mitigated by the ferroptosis inhibitor ferrostatin-1, antioxidants, or by the iron scavenger deferoxamine (DFO). DFO can inhibit ferroptosis by preventing iron ions from supplying electrons to oxygen to form ROS. However, the specific mechanism is still unclear. Dixon et al. made synthetic ferrostatin-1 (ferroptosis inhibitor) and proved that it could specifically inhibit ferroptosis, but it did not impede other oxidative substances and apoptosisinduced death (21). Yao et al. found that intra-articular injection of ferrostatin-1 increased the expression of collagen II, promoted the activation of the Nrf2 antioxidant system, and reduced cartilage degradation, which is beneficial to alleviate joint inflammation (39). Some natural polyphenol compounds can also significantly inhibit ferroptosis, such as baicalein, curcumin, and gastrodin $(73,74)$. Baicalein was demonstrated to suppress $\mathrm{T}$ cell proliferation in collagen-induced arthritis model mice and significantly improve T cell-mediated autoimmune diseases (75). Studies have confirmed that curcumin alleviates inflammation, synovial hyperplasia, and the other main features involved in the pathogenesis of collageninduced arthritis (76). Targeting ferroptosis regulators may be a new direction for developing therapeutic drugs for RA.

\section{DISCUSSION}

In summary, ferroptosis is a recently discovered significant regulatory cell death pattern, and three protective pathways have been successively confirmed. An in-depth study of the underlying mechanism of ferroptosis is of great significance for mapping its role in various related autoimmune diseases. It is worth noting that different cell types (synovial cells, chondrocytes, osteoclasts, and macrophages) may have different susceptibility to ferroptosis. The expression profile of specific genes related to the ferroptosis pathway may not be observed in all cells. Moreover, attention should be paid to the phenomenon of ferroptosis, and the judgment criteria may vary depending on the trigger mechanism. Herein, it is necessary to study

\section{REFERENCES}

1. Philippou E, Petersson SD, Rodomar C, Nikiphorou E. Rheumatoid Arthritis and Dietary Interventions: Systematic Review of Clinical Trials. Nutr Rev (2021) 79(4):410-28. doi: 10.1093/nutrit/nuaa033

2. Smolen JS, Aletaha D, McInnes IB. Rheumatoid Arthritis. Lancet (2016) 388 (10055):2023-38. doi: 10.1016/s0140-6736(16)30173-8 the mechanism of ferroptosis regulators. At present, the inner link between ferroptosis and RA has not been studied in depth. With an in-depth understanding of the relationship between ferroptosis and other biological processes, people will find that ferroptosis, apoptosis, autophagy, and other cell death patterns have some common characteristics in their regulations. Although simultaneous regulation of multiple cell death pathways is vital for the treatment of RA, the relationship between these different types of cell death is not yet fully elucidated. This requires further exploration to validate whether they are integrated into a complex regulatory network.

In short, with the unprecedented prosperity of research on ferroptosis, ferroptosis regulation represents a potential future avenue of investigation in the effort to identify novel therapeutic targets for RA.

\section{AUTHOR CONTRIBUTIONS}

TZ wrote the manuscript. DQ revised the manuscript. ZFL raised the idea for the article. YX, ZX, QY, JS, and ZML performed the literature search and data analysis. All authors contributed to the article and approved the submitted version.

\section{FUNDING}

National Natural Science Foundation of China (31960178, 81960863, 81960870); Construction Project of National Traditional Chinese Medicine Clinical Research Base (2018 No. 131); Yunnan Provincial Fund for Medical Research Center: Clinical Evaluation and Basic Research on the Treatment of rheumatoid arthritis and gout by Traditional Chinese medicine (202102AA310006); Clinical Trial for the Treatment of Rheumatoid Arthritis with Warming yang and Smoothening Meridians (201507001-07, registration number: ChiCTR-INR16010290); Clinical Cooperative Project of Chinese and Western Medicine for Major and Knotty Diseases; Yunnan Provincial Key Laboratory Construction Project Funding; Yunnan Provincial Key Laboratory of Chinese Medicine Rheumatology and Immunology; Yunnan Provincial "Ten Thousands Program" Famous Doctor Special; Yunnan Province Qingguo Wang Expert Workstation Construction Project (202005AF150017); Yunnan Applied Basic Research Projects-Union Foundation (2019FF002(-031)); Applied Basic Research Programs of Science and Technology Commission Foundation of Yunnan Province (2019FA007); Scientific Research Fund Project of Yunnan Provincial Department of Education (2021Y461).

3. Wright HL, Moots RJ, Edwards SW. The Multifactorial Role of Neutrophils in Rheumatoid Arthritis. Nat Rev Rheumatol (2014) 10(10):593-601. doi: 10.1038/nrrheum.2014.80

4. Wang W, Li Z, Meng Q, Zhang P, Yan P, Zhang Z, et al. Chronic Calcium Channel Inhibitor Verapamil Antagonizes TNF- $\alpha$-Mediated Inflammatory Reaction and Protects Against Inflammatory Arthritis in Mice. Inflammation (2016) 39(5):1624-34. doi: 10.1007/s10753-016-0396-1 
5. van Delft MAM, Huizinga TWJ. An Overview of Autoantibodies in Rheumatoid Arthritis. J Autoimmun (2020) 110:102392. doi: 10.1016/ j.jaut.2019.102392

6. Tang W, Lu Y, Tian QY, Zhang Y, Guo FJ, Liu GY, et al. The Growth Factor Progranulin Binds to TNF Receptors and is Therapeutic Against Inflammatory Arthritis in Mice. Science (2011) 332(6028):478-84. doi: 10.1126/science.1199214

7. Rubbert-Roth A, Szabó MZ, Kedves M, Nagy G, Atzeni F, Sarzi-Puttini P. Failure of Anti-TNF Treatment in Patients With Rheumatoid Arthritis: The Pros and Cons of the Early Use of Alternative Biological Agents. Autoimmun $\operatorname{Rev}(2019)$ 18(12):102398. doi: 10.1016/j.autrev.2019.102398

8. Ramiro S, Gaujoux-Viala C, Nam JL, Smolen JS, Buch M, Gossec L, et al. Safety of Synthetic and Biological DMARDs: A Systematic Literature Review Informing the 2013 Update of the EULAR Recommendations for Management of Rheumatoid Arthritis. Ann Rheum Dis (2014) 73(3):52935. doi: 10.1136/annrheumdis-2013-204575

9. Strand V, Greenberg JD, Griffith J, Bao Y, Saunders KC, Garg V, et al. Impact of Treatment With Biologic Agents on the Use of Mechanical Devices Among Rheumatoid Arthritis Patients in a Large US Patient Registry. Arthritis Care Res (Hoboken) (2016) 68(7):914-21. doi: 10.1002/acr.22784

10. Chandrupatla D, Molthoff CFM, Lammertsma AA, van der Laken CJ, Jansen G. The Folate Receptor $\beta$ as a Macrophage-Mediated Imaging and Therapeutic Target in Rheumatoid Arthritis. Drug Deliv Transl Res (2019) 9 (1):366-78. doi: 10.1007/s13346-018-0589-2

11. Mahoney-Sánchez L, Bouchaoui H, Ayton S, Devos D, Duce JA, Devedjian JC. Ferroptosis and its Potential Role in the Physiopathology of Parkinson's Disease. Prog Neurobiol (2021) 196:101890. doi: 10.1016/j.pneurobio. 2020.101890

12. Guan X, Li Z, Zhu S, Cheng M, Ju Y, Ren L, et al. Galangin Attenuated Cerebral Ischemia-Reperfusion Injury by Inhibition of Ferroptosis Through Activating the SLC7A11/GPX4 Axis in Gerbils. Life Sci (2021) 264:118660. doi: $10.1016 /$ j.lfs.2020.118660

13. Li P, Jiang M, Li K, Li H, Zhou Y, Xiao X, et al. Glutathione Peroxidase 4Regulated Neutrophil Ferroptosis Induces Systemic Autoimmunity. Nat Immunol (2021) 22(9):1107-17. doi: 10.1038/s41590-021-00993-3

14. Mao H, Zhao Y, Li H, Lei L. Ferroptosis as an Emerging Target in Inflammatory Diseases. Prog Biophys Mol Biol (2020) 155:20-8. doi: 10.1016/j.pbiomolbio.2020.04.001

15. Yu Y, Jiang L, Wang H, Shen Z, Cheng Q, Zhang P, et al. Hepatic Transferrin Plays a Role in Systemic Iron Homeostasis and Liver Ferroptosis. Blood (2020) 136(6):726-39. doi: 10.1182/blood.2019002907

16. Tarp U. Selenium and the Selenium-Dependent Glutathione Peroxidase in Rheumatoid Arthritis. Dan Med Bull (1994) 41(3):264-74. doi: 10.1111/ j.1445-5994.1994.tb02192.x

17. Luo H, Zhang R. Icariin Enhances Cell Survival in LipopolysaccharideInduced Synoviocytes by Suppressing Ferroptosis via the Xc-/GPX4 Axis. Exp Ther Med (2021) 21(1):72. doi: 10.3892/etm.2020.9504

18. Elmore S. Apoptosis: A Review of Programmed Cell Death. Toxicol Pathol (2007) 35(4):495-516. doi: 10.1080/01926230701320337

19. Proskuryakov SY, Gabai VL. Mechanisms of Tumor Cell Necrosis. Curr Pharm Des (2010) 16(1):56-68. doi: 10.2174/138161210789941793

20. Taylor RC, Cullen SP, Martin SJ. Apoptosis: Controlled Demolition at the Cellular Level. Nat Rev Mol Cell Biol (2008) 9(3):231-41. doi: 10.1038/ nrm2312

21. Dixon SJ, Lemberg KM, Lamprecht MR, Skouta R, Zaitsev EM, Gleason CE, et al. Ferroptosis: An Iron-Dependent Form of Nonapoptotic Cell Death. Cell (2012) 149(5):1060-72. doi: 10.1016/j.cell.2012.03.042

22. Yang WS, Stockwell BR. Synthetic Lethal Screening Identifies Compounds Activating Iron-Dependent, Nonapoptotic Cell Death in Oncogenic-RASHarboring Cancer Cells. Chem Biol (2008) 15(3):234-45. doi: 10.1016/ j.chembiol.2008.02.010

23. Stockwell BR, Friedmann Angeli JP, Bayir H, Bush AI, Conrad M, Dixon SJ, et al. Ferroptosis: A Regulated Cell Death Nexus Linking Metabolism, Redox Biology, and Disease. Cell (2017) 171(2):273-85. doi: 10.1016/j.cell. 2017.09.021

24. Yang WS, Stockwell BR. Ferroptosis: Death by Lipid Peroxidation. Trends Cell Biol (2016) 26(3):165-76. doi: 10.1016/j.tcb.2015.10.014
25. Yagoda N, von Rechenberg M, Zaganjor E, Bauer AJ, Yang WS, Fridman DJ, et al. RAS-RAF-MEK-Dependent Oxidative Cell Death Involving VoltageDependent Anion Channels. Nature (2007) 447(7146):864-8. doi: 10.1038/ nature05859

26. Magtanong L, Ko PJ, To M, Cao JY, Forcina GC, Tarangelo A, et al. Exogenous Monounsaturated Fatty Acids Promote a Ferroptosis-Resistant Cell State. Cell Chem Biol (2019) 26(3):420-32.e9. doi: 10.1016/ j.chembiol.2018.11.016

27. Zilka O, Shah R, Li B, Friedmann Angeli JP, Griesser M, Conrad M, et al. On the Mechanism of Cytoprotection by Ferrostatin-1 and Liproxstatin-1 and the Role of Lipid Peroxidation in Ferroptotic Cell Death. ACS Cent Sci (2017) 3 (3):232-43. doi: 10.1021/acscentsci.7b00028

28. Fuhrmann DC, Mondorf A, Beifuß J, Jung M, Brüne B. Hypoxia Inhibits Ferritinophagy, Increases Mitochondrial Ferritin, and Protects From Ferroptosis. Redox Biol (2020) 36:101670. doi: 10.1016/j.redox.2020.101670

29. Jing X, Du T, Chen K, Guo J, Xiang W, Yao X, et al. Icariin Protects Against Iron Overload-Induced Bone Loss via Suppressing Oxidative Stress. J Cell Physiol (2019) 234(7):10123-37. doi: 10.1002/jcp.27678

30. Cen WJ, Feng Y, Li SS, Huang LW, Zhang T, Zhang W, et al. Iron Overload Induces G1 Phase Arrest and Autophagy in Murine Preosteoblast Cells. J Cell Physiol (2018) 233(9):6779-89. doi: 10.1002/jcp.26405

31. Xiao W, Beibei F, Guangsi S, Yu J, Wen Z, Xi H, et al. Iron Overload Increases Osteoclastogenesis and Aggravates the Effects of Ovariectomy on Bone Mass. J Endocrinol (2015) 226(3):121-34. doi: 10.1530/joe-14-0657

32. Hakobyan N, Kazarian T, Jabbar AA, Jabbar KJ, Valentino LA. Pathobiology of Hemophilic Synovitis I: Overexpression of Mdm2 Oncogene. Blood (2004) 104(7):2060-4. doi: 10.1182/blood-2003-12-4231

33. Fritz P, Saal JG, Wicherek C, König A, Laschner W, Rautenstrauch H. Quantitative Photometrical Assessment of Iron Deposits in Synovial Membranes in Different Joint Diseases. Rheumatol Int (1996) 15(5):211-6. doi: 10.1007/bf00290523

34. Ogilvie-Harris DJ, Fornaiser VL. Synovial Iron Deposition in Osteoarthritis and Rheumatoid Arthritis. J Rheumatol (1980) 7(1):30-6.

35. Tański W, Chabowski M, Jankowska-Polańska B, Jankowska EA. Iron Metabolism in Patients With Rheumatoid Arthritis. Eur Rev Med Pharmacol Sci (2021) 25(12):4325-35. doi: 10.26355/eurrev_202106_26140

36. Ma XN, Zhou J, Ge BF, Zhen P, Ma HP, Shi WG, et al. Icariin Induces Osteoblast Differentiation and Mineralization Without Dexamethasone In Vitro. Planta Med (2013) 79(16):1501-8. doi: 10.1055/s-0033-1350802

37. Basit F, van Oppen LM, Schöckel L, Bossenbroek HM, van Emst-de Vries SE, Hermeling JC, et al. Mitochondrial Complex I Inhibition Triggers a Mitophagy-Dependent ROS Increase Leading to Necroptosis and Ferroptosis in Melanoma Cells. Cell Death Dis (2017) 8(3):e2716. doi: $10.1038 /$ cddis.2017.133

38. Dixon SJ, Stockwell BR. The Role of Iron and Reactive Oxygen Species in Cell Death. Nat Chem Biol (2014) 10(1):9-17. doi: 10.1038/nchembio.1416

39. Yao X, Sun K, Yu S, Luo J, Guo J, Lin J, et al. Chondrocyte Ferroptosis Contribute to the Progression of Osteoarthritis. J Orthop Translat (2021) 27:33-43. doi: 10.1016/j.jot.2020.09.006

40. Bersuker K, Hendricks JM, Li Z, Magtanong L, Ford B, Tang PH, et al. The CoQ Oxidoreductase FSP1 Acts Parallel to GPX4 to Inhibit Ferroptosis. Nature (2019) 575(7784):688-92. doi: 10.1038/s41586-019-1705-2

41. Xie Z, Hou H, Luo D, An R, Zhao Y, Qiu C. ROS-Dependent Lipid Peroxidation and Reliant Antioxidant Ferroptosis-Suppressor-Protein 1 in Rheumatoid Arthritis: A Covert Clue for Potential Therapy. Inflammation (2021) 44(1):35-47. doi: 10.1007/s10753-020-01338-2

42. Doll S, Freitas FP, Shah R, Aldrovandi M, da Silva MC, Ingold I, et al. FSP1 is a Glutathione-Independent Ferroptosis Suppressor. Nature (2019) 575 (7784):693-8. doi: 10.1038/s41586-019-1707-0

43. Kraft VAN, Bezjian CT, Pfeiffer S, Ringelstetter L, Müller C, Zandkarimi F, et al. GTP Cyclohydrolase 1/Tetrahydrobiopterin Counteract Ferroptosis Through Lipid Remodeling. ACS Cent Sci (2020) 6(1):41-53. doi: 10.1021/ acscentsci.9b01063

44. Datta S, Kundu S, Ghosh P, De S, Ghosh A, Chatterjee M. Correlation of Oxidant Status With Oxidative Tissue Damage in Patients With Rheumatoid Arthritis. Clin Rheumatol (2014) 33(11):1557-64. doi: 10.1007/s10067-014$2597-\mathrm{z}$ 
45. Su LJ, Zhang JH, Gomez H, Murugan R, Hong X, Xu D, et al. Reactive Oxygen Species-Induced Lipid Peroxidation in Apoptosis, Autophagy, and Ferroptosis. Oxid Med Cell Longev (2019) 2019:5080843. doi: 10.1155/2019/5080843

46. Phull AR, Nasir B, Haq IU, Kim SJ. Oxidative Stress, Consequences and ROS Mediated Cellular Signaling in Rheumatoid Arthritis. Chem Biol Interact (2018) 281:121-36. doi: 10.1016/j.cbi.2017.12.024

47. Quiñonez-Flores CM, González-Chávez SA, Del Río Nájera D, Pacheco-Tena C. Oxidative Stress Relevance in the Pathogenesis of the Rheumatoid Arthritis: A Systematic Review. BioMed Res Int (2016) 2016:6097417. doi: 10.1155/ 2016/6097417

48. Mateen S, Moin S, Khan AQ, Zafar A, Fatima N. Increased Reactive Oxygen Species Formation and Oxidative Stress in Rheumatoid Arthritis. PLoS One (2016) 11(4):e0152925. doi: 10.1371/journal.pone.0152925

49. Sareila O, Kelkka T, Pizzolla A, Hultqvist M, Holmdahl R. NOX2 ComplexDerived ROS as Immune Regulators. Antioxid Redox Signal (2011) 15 (8):2197-208. doi: 10.1089/ars.2010.3635

50. Lee K, Won HY, Bae MA, Hong JH, Hwang ES. Spontaneous and AgingDependent Development of Arthritis in NADPH Oxidase 2 Deficiency Through Altered Differentiation of CD11b+ and Th/Treg Cells. Proc Natl Acad Sci USA (2011) 108(23):9548-53. doi: 10.1073/pnas.1012645108

51. Ehrenstein MR, Evans JG, Singh A, Moore S, Warnes G, Isenberg DA, et al. Compromised Function of Regulatory T Cells in Rheumatoid Arthritis and Reversal by Anti-TNFalpha Therapy. J Exp Med (2004) 200(3):277-85. doi: $10.1084 /$ jem.20040165

52. Möttönen M, Heikkinen J, Mustonen L, Isomäki P, Luukkainen R, Lassila O. CD4+ CD25+ T Cells With the Phenotypic and Functional Characteristics of Regulatory T Cells are Enriched in the Synovial Fluid of Patients With Rheumatoid Arthritis. Clin Exp Immunol (2005) 140(2):360-7. doi: 10.1111/ j.1365-2249.2005.02754.x

53. Yang M, Haase C, Viljanen J, Xu B, Ge C, Kihlberg J, et al. Cutting Edge: Processing of Oxidized Peptides in Macrophages Regulates T Cell Activation and Development of Autoimmune Arthritis. J Immunol (2017) 199(12):393742. doi: 10.4049/jimmunol.1700774

54. Telfer JF, Brock JH. Proinflammatory Cytokines Increase Iron Uptake Into Human Monocytes and Synovial Fibroblasts From Patients With Rheumatoid Arthritis. Med Sci Monit (2004) 10(4):Br91-5.

55. Valentino LA. Blood-Induced Joint Disease: The Pathophysiology of Hemophilic Arthropathy. J Thromb Haemost (2010) 8(9):1895-902. doi: $10.1111 / \mathrm{j} .1538-7836.2010 .03962 . x$

56. Melchiorre D, Manetti M, Matucci-Cerinic M. Pathophysiology of Hemophilic Arthropathy. J Clin Med (2017) 6(7):63. doi: 10.3390/jcm6070063

57. Sun Y, Cheung HS. P53, Proto-Oncogene and Rheumatoid Arthritis. Semin Arthritis Rheum (2002) 31(5):299-310. doi: 10.1053/sarh.2002.31550

58. Takatori H, Kawashima H, Suzuki K, Nakajima H. Role of P53 in Systemic Autoimmune Diseases. Crit Rev Immunol (2014) 34(6):509-16. doi: 10.1615/ critrevimmunol.2014012193

59. Xu M, Feng M, Peng H, Qian Z, Zhao L, Wu S. Epigenetic Regulation of Chondrocyte Hypertrophy and Apoptosis Through Sirt1/P53/P21 Pathway in Surgery-Induced Osteoarthritis. Biochem Biophys Res Commun (2020) 528 (1):179-85. doi: 10.1016/j.bbrc.2020.04.097

60. Migita K, Tanaka F, Yamasaki S, Shibatomi K, Ida H, Kawakami A, et al. Regulation of Rheumatoid Synoviocyte Proliferation by Endogenous P53 Induction. Clin Exp Immunol (2001) 126(2):334-8. doi: 10.1046/j.13652249.2001.01677.x

61. Park JS, Lim MA, Cho ML, Ryu JG, Moon YM, Jhun JY, et al. P53 Controls Autoimmune Arthritis via STAT-Mediated Regulation of the Th17 Cell/Treg Cell Balance in Mice. Arthritis Rheum (2013) 65(4):949-59. doi: 10.1002/art.37841

62. Maas K, Westfall M, Pietenpol J, Olsen NJ, Aune T. Reduced P53 in Peripheral Blood Mononuclear Cells From Patients With Rheumatoid Arthritis is Associated With Loss of Radiation-Induced Apoptosis. Arthritis Rheum (2005) 52(4):1047-57. doi: 10.1002/art.20931

63. Doll S, Proneth B, Tyurina YY, Panzilius E, Kobayashi S, Ingold I, et al. ACSL4 Dictates Ferroptosis Sensitivity by Shaping Cellular Lipid Composition. Nat Chem Biol (2017) 13(1):91-8. doi: 10.1038/nchembio.2239
64. Kang MJ, Fujino T, Sasano H, Minekura H, Yabuki N, Nagura H, et al. A Novel Arachidonate-Preferring Acyl-CoA Synthetase is Present in Steroidogenic Cells of the Rat Adrenal, Ovary, and Testis. Proc Natl Acad Sci USA (1997) 94(7):2880-4. doi: 10.1073/pnas.94.7.2880

65. Yang XY, Zheng KD, Lin K, Zheng G, Zou H, Wang JM, et al. Energy Metabolism Disorder as a Contributing Factor of Rheumatoid Arthritis: A Comparative Proteomic and Metabolomic Study. PLoS One (2015) 10(7): e0132695. doi: 10.1371/journal.pone.0132695

66. Wang S, Deng Z, Ma Y, Jin J, Qi F, Li S, et al. The Role of Autophagy and Mitophagy in Bone Metabolic Disorders. Int J Biol Sci (2020) 16(14):2675-91. doi: $10.7150 /$ ijbs. 46627

67. Jhun J, Moon J, Ryu J, Shin Y, Lee S, Cho KH, et al. Liposome/gold Hybrid Nanoparticle Encoded With CoQ10 (LGNP-CoQ10) Suppressed Rheumatoid Arthritis via STAT3/Th17 Targeting. PLoS One (2020) 15(11):e0241080. doi: 10.1371/journal.pone.0241080

68. Dodson M, Castro-Portuguez R, Zhang DD. NRF2 Plays a Critical Role in Mitigating Lipid Peroxidation and Ferroptosis. Redox Biol (2019) 23:101107. doi: 10.1016/j.redox.2019.101107

69. Chadha S, Behl T, Kumar A, Khullar G, Arora S. Role of Nrf2 in Rheumatoid Arthritis. Curr Res Transl Med (2020) 68(4):171-81. doi: 10.1016/j.retram. 2020.05.002

70. Zhang Y, Wang G, Wang T, Cao W, Zhang L, Chen X. Nrf2-Keap1 PathwayMediated Effects of Resveratrol on Oxidative Stress and Apoptosis in Hydrogen Peroxide-Treated Rheumatoid Arthritis Fibroblast-Like Synoviocytes. Ann N Y Acad Sci (2019) 1457(1):166-78. doi: 10.1111/ nyas. 14196

71. Alcaraz MJ, Ferrándiz ML. Relevance of Nrf2 and Heme Oxygenase-1 in Articular Diseases. Free Radic Biol Med (2020) 157:83-93. doi: 10.1016/ j.freeradbiomed.2019.12.007

72. Chang LC, Chiang SK, Chen SE, Yu YL, Chou RH, Chang WC. Heme Oxygenase-1 Mediates BAY 11-7085 Induced Ferroptosis. Cancer Lett (2018) 416:124-37. doi: 10.1016/j.canlet.2017.12.025

73. Kose T, Vera-Aviles M, Sharp PA, Latunde-Dada GO. Curcumin and (-)Epigallocatechin-3-Gallate Protect Murine MIN6 Pancreatic Beta-Cells Against Iron Toxicity and Erastin-Induced Ferroptosis. Pharmaceuticals (Basel) (2019) 12(1):26. doi: 10.3390/ph12010026

74. Jiang T, Cheng H, Su J, Wang X, Wang Q, Chu J, et al. Gastrodin Protects Against Glutamate-Induced Ferroptosis in HT-22 Cells Through Nrf2/HO-1 Signaling Pathway. Toxicol Vitro (2020) 62:104715. doi: 10.1016/ j.tiv.2019.104715

75. Xu J, Liu J, Yue G, Sun M, Li J, Xiu X, et al. Therapeutic Effect of the Natural Compounds Baicalein and Baicalin on Autoimmune Diseases. Mol Med Rep (2018) 18(1):1149-54. doi: 10.3892/mmr.2018.9054

76. Dai Q, Zhou D, Xu L, Song X. Curcumin Alleviates Rheumatoid ArthritisInduced Inflammation and Synovial Hyperplasia by Targeting mTOR Pathway in Rats. Drug Des Devel Ther (2018) 12:4095-105. doi: 10.2147/ dddt.S175763

Conflict of Interest: The authors declare that the research was conducted in the absence of any commercial or financial relationships that could be construed as a potential conflict of interest.

Publisher's Note: All claims expressed in this article are solely those of the authors and do not necessarily represent those of their affiliated organizations, or those of the publisher, the editors and the reviewers. Any product that may be evaluated in this article, or claim that may be made by its manufacturer, is not guaranteed or endorsed by the publisher.

Copyright $\odot 2022$ Zhao, Yang, Xi, Xie, Shen, Li, Li and Qin. This is an open-access article distributed under the terms of the Creative Commons Attribution License (CC BY). The use, distribution or reproduction in other forums is permitted, provided the original author(s) and the copyright owner(s) are credited and that the original publication in this journal is cited, in accordance with accepted academic practice. No use, distribution or reproduction is permitted which does not comply with these terms. 